\title{
Start-up Optimization of a Combined Cycle Power Plant
}

\author{
A. Lind ${ }^{a}, \quad$ E. Sällberg ${ }^{a}$, \\ S. Velut ${ }^{b}, \mathrm{~S}$. Gallardo Yances ${ }^{c}, \mathrm{~J} . \AA_{\text {Akesson }}^{a}{ }^{b}, \mathrm{~K}$. Link $^{c}$ \\ ${ }^{a}$ Lund University, Department of Automatic Control, Lund, Sweden \\ ${ }^{b}$ Modelon AB, Lund, Sweden \\ ${ }^{c}$ Siemens AG, Energy Sector, Erlangen, Germany
}

\section{Abstract}

In the electricity market of today, with increasing demand for electricity production on short notice, the combined cycle power plant stands high regarding fast start-ups and efficiency. In this paper, it has been shown how the dynamic start-up procedure of a combined cycle power plant can be optimized using direct collocation methods, proposing a way to minimize the start-up time while maximizing the power production during start-up. Physical models derived from first principles have been developed in Modelica specifically for optimization purposes, in that the models contain no discontinuities. Also, the models used for optimization are simpler than typical highfidelity simulation models. Two different models used for optimization in four different start-up scenarios are presented in the paper. A critically limiting factor during start-up is the stress of important components, e.g., the evaporator. In order to take this aspect into account, constraints on the stress levels of such components have been introduced in the optimization formulation. In particular, it is shown how a pressure dependent stress constraint, similar to what is used in actual operation, can be applied in optimization. Also, different assumptions about which control variables to optimize are explored. Results are encouraging and show that energy production during start-up can be significantly increased by increasing the number of control inputs available to the optimizer, while maintaining desirable lifetime of critical components by introducing constrains on acceptable stress levels.

Keywords: Combined Cycle Power Plants, Start-up, Dynamic optimization, Optimica, Control, Modelica, Modeling

\section{Introduction}

In a time when the production from renewable energy sources is steadily growing the demand for complementary electricity production on short notice is high. Large fluctuations during the day require power generators to react quickly to maintain the balance between demand and production. Deregulation of the electric power market also allows private investors to install power plants and supply power to the grid, which has increased the competition on the electricity market. The requirements between demand and supply have to be maintained while offering electricity at the lowest cost.

When considering fast start-ups and efficiency, the combined cycle power plant stands high in comparison with other electricity production methods. In this paper, the start-up procedure of a combined cycle power plant is studied. The aim is to minimize the start-up time while keeping the lifetime consumption of crucial power plant components under control and maximizing the amount of power output produced.

Several previous studies that deal with optimization of the start-up of combined cycle power plants have been made. In Casella and Pretolani, [1], optimization with a trial-and-error method is presented where the results are obtained by simulating Modelica power plant models. The study has been carried out to develop simplified models that can be used to automatically compute the optimal transients with an optimization software and the models were based on the Modelica ThermoPower library, see Casella and Leva, [2]. A model-based approach for optimizing the gas turbine load trajectory has been studied in Casella et al., [3]. A simplified model is developed based on interpolated locally identified linear models and the procedure aims at deriving the gas turbine load profile described by a parameterized function. A minimumtime problem is solved to determine the parameters 
of the parameterized function. In [4] a combined cycle power plant is modeled and optimized, where the thermo-mechanical stress in the steam turbine rotor is considered as the most limiting factor during the start-up. Shirakawa et al. proposed an optimal design method combining dynamic simulation and nonlinear programming in [5].

The aim of the current paper is to make the startup procedure of a combined cycle power plant more efficient, with respect to the start-up time and power production, while limiting the thermal stress in the heat recovery steam generator. The plant models are described in the object-oriented modeling language Modelica. All models are developed by Siemens AG, Energy Sector, in cooperation with Modelon AB, and are based on elementary models from first principle equations of mass and energy. The physical models have been developed using the commercial Modelica simulation environment, Dymola [6] and they have been adapted to suit optimization purposes. The tool used for optimization is the Modelica based open source platform JModelica.org.

The paper is structured as follows: Section 2 gives some background information about combined cycle power plants, dynamic optimization, JModelica.org and Optimica, while Section 3 describes the power plant model. Section 4 presents the optimal start-up problem formulation and the numerical results are discussed. Section 5 summarizes the results of this paper and gives proposals for future work.

\section{Background}

\subsection{Combined Cycle Power Plants}

The basic principle of a combined cycle power plant (CCPP) is to combine two thermal cycles in one power plant, where the topping cycle is a cycle operating at a higher temperature and the bottoming cycle is a cycle operating at a lower temperature level. The waste heat that the topping cycle produces is used in the process of the bottoming cycle and the efficiency is higher for the combined cycle than that of one cycle alone. In the commercial power generation of today the combined cycle power plants consist of a gas topping cycle and a steam/water bottoming cycle [7].

The plant is constructed mainly with three parts, the gas turbine (GT), the heat recovery steam generator (HRSG) and the steam turbine (ST).

In the GT, ambient air is drawn into the turbine, compressed and used to burn some combustion medium. Hot gas is produced and expands in the turbine where it is used to drive both the compressor and the generator.

The key component of a CCPP is the HRSG which couples the two cycles so that the heat from the GT exhaust gas is used to produce hot steam which drives the ST. The HRSG consists mainly of three components; the economizer, the evaporator and the superheater. The water is preheated in the economizer, evaporated to wet steam in the evaporator and the steam is dried in the superheater. When the steam is of high enough quality it is expanded in the ST where it generates power.

The net efficiency can reach more than $60 \%$ in today's CCPPs. About $60-70 \%$ of the total power output is produced in the GT [7].

The start-up of a CCPP is normally scheduled as follows:

1 The GT is first accelerated to full speed no load and it is synchronized to the grid.

2 The load of the GT is increased and the boiler starts producing steam. The generated steam is not led to the ST but bypassed to a condenser.

3 When the steam quality is high enough, the bypass valve is slowly closed and the steam can drive the ST.

Reducing the start-up time of the CCPP is typically achieved by maximizing the loading rates of both turbines while maintaining the lifetime consumption of critically stressed components under control. One of the critical components is the drum in the evaporator. During the second phase of the start-up, the walls of this component are subject to high thermal stress due to temperature gradient transients. The ST is also subject to large stress constraints, but this occurs in the last phase of the start-up. The paper focuses on the optimization of the second phase, that is the loading of the GT.

\subsection{The Dynamic Optimization Problem}

The start-up optimization of the CCPP has been formulated as a dynamic optimization problem. The optimization consists typically in finding time trajectories of the control variables, $u(t)$, that minimize an objective function $\varphi$ expressed in terms of process variables $y$. The optimization problem can generally be stated as:

$$
\min _{u(t)} \varphi\left(z(t), y(t), u(t), t_{f}\right)
$$


subject to

$$
\begin{array}{r}
\frac{d z(t)}{d t}=F(z(t), y(t), u(t), t) \\
0=G(z(t), y(t), u(t), t) \\
z(0)=z^{0}
\end{array}
$$

with the bounds

$$
\begin{gathered}
z^{L} \leq z(t) \leq z^{U} \\
y^{L} \leq y(t) \leq y^{U} \\
u^{L} \leq u(t) \leq u^{U} \\
t_{f}^{L} \leq t_{f} \leq t_{f}^{U}
\end{gathered}
$$

where

$\varphi$ is a scalar objective function,

$F$ are the right hand sides of differential equation constraints,

$G$ are algebraic equation constraints, assumed to be index one,

$z$ are differential state profile vectors,

$z_{0}$ are the initial values of $z$

$y$ are algebraic state profile vectors,

$u$ are control profile vectors,

$t_{f}$ is the final time.[8]

The objective function $\varphi$, that is to be minimized, can have multiple forms; one is given by the Lagrange form:

$$
\varphi=\int_{t_{0}}^{t_{f}} L(z(t), y(t), u(t), t) d t
$$

\subsection{JModelica.org}

In this project, the tool used for optimization is the open source platform JModelica.org [9].

JModelica.org is an extensible Modelica-based open source platform for optimization, simulation and analysis of complex dynamic systems. The main objective of the project is to create an industrially viable open source platform for optimization of Modelica models, while offering a flexible platform serving as a virtual lab for algorithm development and research. [9]

JModelica.org offers different types of model objects that can be used for simulation and optimization. For simulation purposes, a Functional Mock-up Unit (FMU) that follows the FMI (Functional Mock-up Interface) standard, is used. It is created by compiling a Modelica model in JModelica.org or in any other tool which supports the FMU export. The FMU file is thereafter loaded as an FMUModel Python object in JModelica.org and can be simulated using the Assimulo package. For a more detailed description of import and export of FMUs in python, see [10]. For optimization purposes a JMUModel object is instead created. A JMU is a compressed file following a JModelica.org specific standard that is close to the FMI standard. After compilation, the JMU file is loaded into JModelica.org and the JMUModel is created and can be optimized using state of the art numerical methods. See Åkesson et al. [11] for a thorough description of the JModelica.org platform.

\subsubsection{Collocation Method}

The JModelica.org platform uses a direct collocation method based on Lagrange polynomials on finite elements with Radau points [12]. The Differential Algebraic Equations (DAE) are transformed to a nonlinear program (NLP) by approximating control and state profiles by piecewise polynomial. The NLP problem is solved by the solver IPOPT [8].

\subsubsection{IPOPT}

The open-source software IPOPT (Interior Point OPTimizer) is a package for large-scale nonlinear optimization. The optimization problem is transferred to an interior point problem formulation where a logarithmic barrier term replaces the inequality constraints [13].

\subsection{Optimica}

Optimica is an extension of the Modelica language that enables high-level formulation of optimization problems based on Modelica models. The extension mainly consists of an additional class, optimization, which includes the attribute objective that specifies the objective function of the optimization problem. Another supplement is the constraint section, which can handle different kinds of linear and non-linear equality- and inequality constraints. [14]

\section{Models}

\subsection{Plant Model}

In this paper, three models of a CCPP with different complexities have been considered referred to as CCPP1, CCPP2 and CCPP3, see Figures 1, 2 and 3, respectively. 
All models are developed in Modelica, [15], using the commercial modeling and simulation environment Dymola [6] and are based on elementary models from first principle equations of mass and energy. Discontinuities have been smoothed and all equations are twice continuously differentiable. Components are modeled separately according to the object-oriented principle and joined by additional connection equations to form the complete system model. Some of the components in the Dymola models are not connected by visible connector lines but only by Modelica equations. This is the case for the output of the integrator at the valve opening, which is connected to the real expression at the valve just above it and also the two outputs of the GT which are connected to the two real expressions to the right of the GT.

The water side is modeled by dynamic balance equations whereas the gas side is static. The simplified HRSG model, see Figures 1, 2 and 3, consist of an HP pressure stage boiler and is represented by lumped volume models of a superheater and an evaporator. To attain better accuracy with respect to thermal discretization, the superheater is described by five partial components with different tube geometries. An ideal level control is assumed in the evaporator model and it computes the water/steam flow through the HRSG. The evaporator drum is modeled as a volume, where the wall, which is subject to high stress during transients, is spatially discretized. The GT model computes temperature and mass flow of the gas entering the HRSG at every load. The bypass valve controls the pressure in the water circuit and can be actuated by a pressure controller to limit large pressure transients. A constant pressure has been chosen as boundary condition for the bypass valve, corresponding to the pressure in the condenser. The models CCPP1 and CCPP2 differ in that a pressure controller acting on the bypass valve is introduced in CCPP1, whereas in CCPP2, the bypass valve is used as a manipulated control variables available for optimization.

The model CCPP3, see Figure 3 is more detailed than models CCPP1 and CCPP2 in that it is modeled with an additional IP reheater apart from an HP superheater and an HP evaporator. The reheater is described by three partial components and the superheater has four partial components with different tube geometries which are operating at different pressures like in CCPP1 and CCPP2 as in the simplified model. An additional component that has been added to CCPP3 is the header of the part of the superheater operating at highest temperature, see component Header in Figure
3. The header is in this model considered as a component subject to high stress during start-up transients together with the evaporator drum.

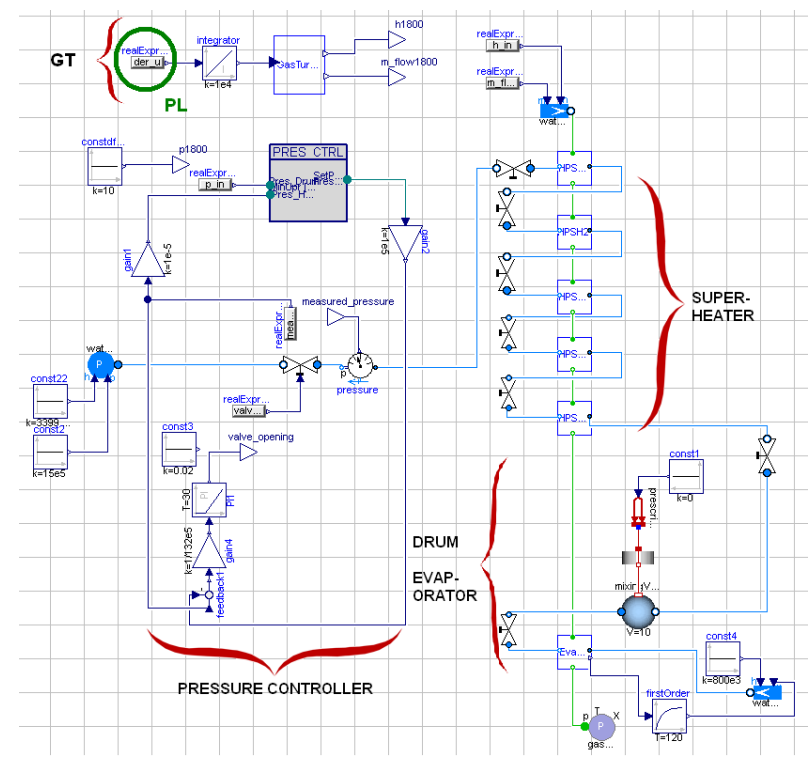

Figure 1: Modelica object diagram of model CCPP1, including a pressure controller. The main components are marked and the degree of freedom PL (Power Load) is circled.

\subsection{Water and Steam Properties}

Pressure and specific enthalpy have been chosen as states in the balance equations on the water side. Correlations to compute temperature as well as density and its derivatives with respect to pressure and enthalpy need therefore to be derived. Polynomial approximations expressed as Taylor expansions from the phase boundaries have been chosen, see [16] for a similar method. This leads to optimization friendly and accurate medium properties and also a continuous transition of temperature and density across the phase boundaries.

\section{GT Load Profile Optimization}

\subsection{Problem Formulation}

The aim of the optimization is to minimize the startup time of the CCPP while keeping the lifetime consumption of critically stressed components under control and maximizing the amount of power output produced. Four different optimization problems are considered, namely, i) a 1DOF problem based on the model CCPP1 is considered, ii) a 2DOF problem 


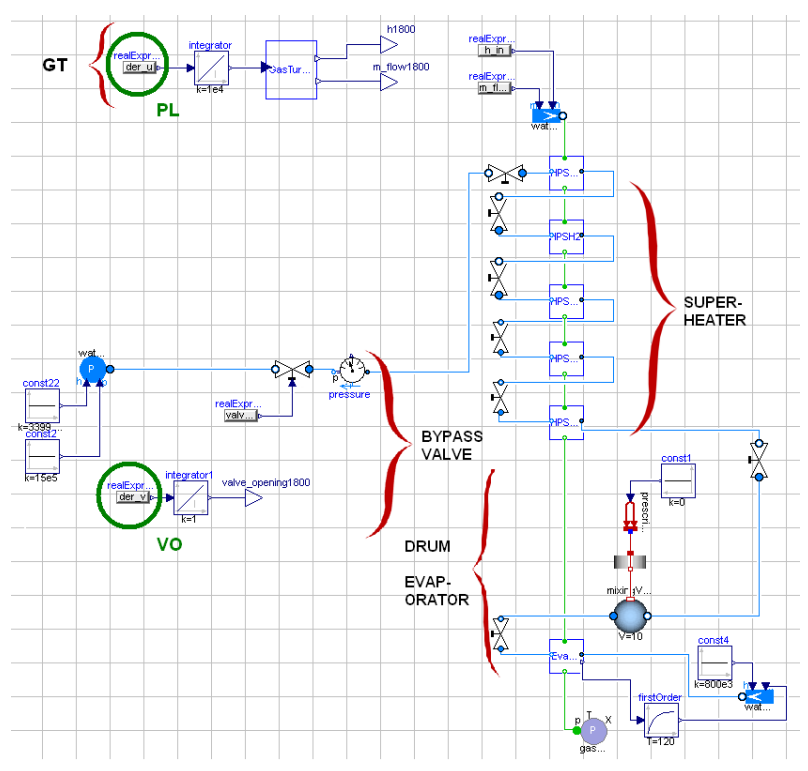

Figure 2: Modelica object diagram of model CCPP2. The main components are marked and the degrees of freedom PL (Power Load) and VO (Valve Opening) are circled.

based on CCPP2, iii) a 2DOF problem with constant thermal stress bounds based on CCPP 3 and finally $i v$ ) a 2DOF problem with pressure dependent thermal stress bounds based on CCPP3.

\subsubsection{Optimization Phase}

The optimization starts after the synchronization of the GT to the grid. The time between stand-still and full-speed-no-load is not subject to optimization but is simulated to compute the initial point of the optimization. In the present study, a hot start is assumed, which means that the start-up is initiated after a standstill time of at most 7 hours. The start-up is considered to be complete when the GT has reached its full load, i.e. its maximum power output.

\subsubsection{Degrees of Freedom}

Two control variables have been considered in the paper: the load $u$ of the GT and the opening $v$ of the bypass valve. The degrees of freedom in the optimization are defined as the time-derivative of the control variables, i.e. $d u / d t$ (marked as PL for Power Load in Figures 1, 2 and 3) and $d v / d t$ (marked as VO for Valve Opening in Figures 2 and 3), and are parameterized by piecewise constant signals.

In a first optimization problem, the GT load $u$ is chosen to be the only control variable. The bypass valve is in that case manipulated by a PI controller to con-

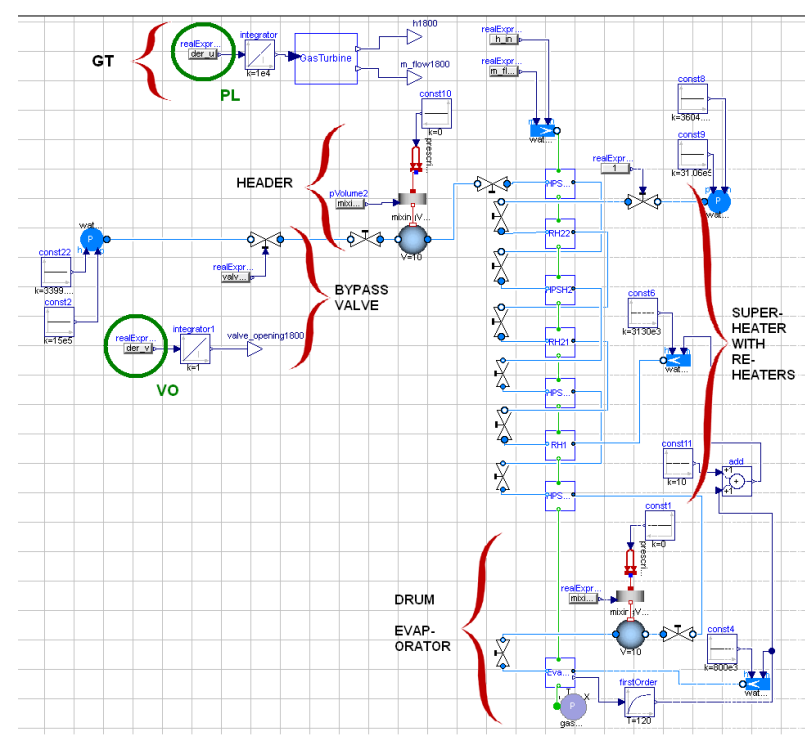

Figure 3: Modelica object diagram of model CCPP3, including a header and an IP reheater. The main components are marked and the degrees of freedom PL (Power Load) and VO (Valve Opening) are circled.

trol the pressure at the superheater outlet, Figures 1. In a second optimization problem, the pressure controller, seen in Figure 1, is removed and both degrees of freedom are used for optimization. In these cases, the physical models of the power plant are identical, apart from the pressure controller. For the two optimization problems based on CCPP3, both degrees of freedom are used.

\subsubsection{Cost Function}

The objective function is written in the Lagrange form as in Equation (9). The optimization problem has been formulated using a quadratic cost function where the integrand $L$ penalizes the deviation of the load $u$ from its reference value $u_{\text {ref }}$ as well as the derivatives of the inputs:

$$
L=\alpha\left(u-u_{r e f}\right)^{2}+\beta \frac{d u^{2}}{d t}+\gamma \frac{d v^{2}}{d t} .
$$

The reference value for $u(t)$ is normalized to 1 , which corresponds to $100 \%$ of its full load. This formulation maximizes the produced power output during start-up and should also result in a short start-up time.

\subsubsection{Constraints}

The limiting factor during the start-up procedure is the thermal stress due to temperature gradient transients in the wall of the drum of the evaporator and superheater header. The simplified optimization constraint 
considered for CCPP1 and CCPP2 is the temperature gradient in the wall of the boiler:

$$
\left|T_{\text {middle layer wallDrum }}-T_{\text {evap }}\right| \leq\left|d T_{\text {maxDrum }}\right|=0.5 \text {. }
$$

In the first optimization problem based on CCPP3, an additional constraint for the temperature gradient in the header is added:

$$
\left|T_{\text {middle layer wallHeader }}-T_{S H}\right| \leq\left|d T_{\text {maxHeader }}\right|=0.5 \text {. }
$$

In the second optimization problem based on CCPP3, the constant bounds on the drum and header temperature gradients are replaced by pressure dependent constraints

$$
\begin{aligned}
& \left|T_{\text {middle layer wallDrum }}-T_{\text {evap }}\right| \leq f_{d}(p) \\
& \left|T_{\text {middle layer wallHeader }}-T_{S H}\right| \leq f_{h}(p) .
\end{aligned}
$$

When the bypass valve opening is used for optimization, an additional constraint on the opening derivative is introduced:

$$
\left|\frac{d v}{d t}\right|<\left|\frac{d v}{d t}\right|_{\max }
$$

\subsubsection{Initialization}

To initialize the first optimization problem, a simulation of the model is first realized in JModelica.org, using a simple (zero-load) input trajectory. This results in feasible trajectories for the optimization that do not violate the defined constraints. The simulation result is then used as an initial guess trajectory for the first optimization. To improve the result accuracy the optimization is done iteratively, starting with a simple discretization with few elements. The result of the previous optimization is then used as a new initial guess trajectory and the discretization is refined by increasing the number of elements and/or by changing the end time of the optimization.

\subsubsection{Optimization Settings}

The number of elements, $n_{e}$, in the optimization interval has been varied between 10 and 45 and the number of collocation points in every element was fixed to $n_{c p}=3$. The overall relative tolerance for the interior point solver was chosen to be $10^{-4}$.

\subsection{DOF Optimization of CCPP1}

The pressure in the HRSG is controlled using the opening $v$ of the bypass valve in a built in controlloop, leaving $u$ as the sole degree of freedom (1
DOF) for optimization. The continuous-time optimization model contains 28 continuous time states and 456 scalar equations. The power output has been allowed to either both increase and decrease during startup (non-monotonic power output) or to only increase (monotonically increasing power output). Both cases have been optimally controlled to full load and the optimization results are shown in Figures 4 and 5. The solid line trajectory represents the solution for the nonmonotonic power output and the dashed trajectory represents the monotonically increasing power output.
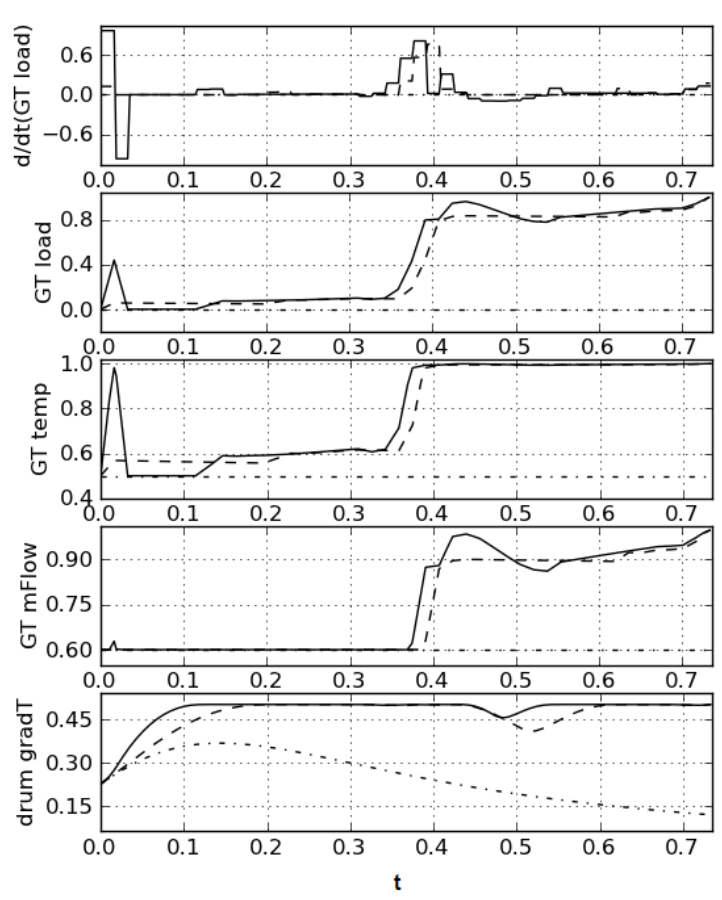

Figure 4: Optimal start-up trajectories for 1 DOF: dashed (monotonically increasing power output) and solid (non-monotonic power output) curves. Simulated initial guess trajectories are shown by dashdotted curves. From the top: the derivative of the GT load, the GT load, the GT outlet temperature, the GT mass flow and the temperature gradient in the wall of the drum. All results and times have been normalized.

The optimal and normalized time for the GT to reach $95 \%$ of full load is approximately the same in both cases: 0.724 and 0.723 for the monotonic and non-monotonic load profile, respectively. From Figure 4 , it can be seen that the temperature gradient constraint becomes rapidly active in spite of the low GT load. This is due to that the pressure controller keeps the bypass valve closed which results in a low mass flow through the valve and a high pressure in the 

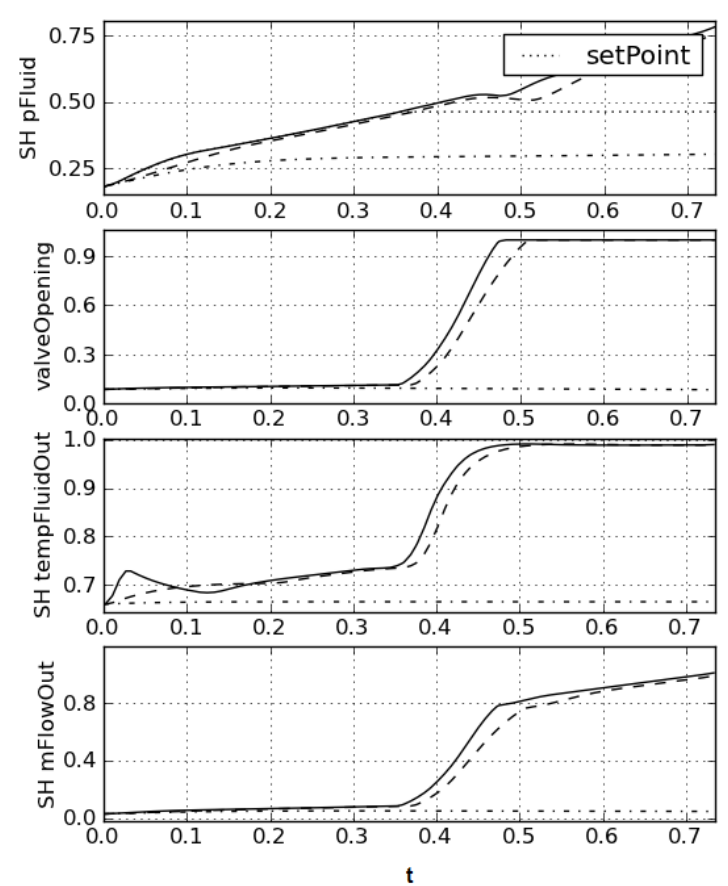

Figure 5: Optimal start-up trajectories for 1 DOF: dashed (monotonically increasing power output) and solid (non-monotonic power output) curves. Simulated initial guess trajectories are shown by dashdotted curves. From the top: the pressure in the superheater on the steam/water side and the pressure control loop set-point, the bypass valve opening in the pressure controller, the outlet temperature and the outlet mass flow from the superheater on the steam/water side. All results and times have been normalized.

HRSG, giving large temperature gradients in the wall of the drum. There is also hot steam in the HRSG due to transients from phase 1 and from the fact that the start-up is considered as a hot start. At about $t=0.37$, the GT load is rapidly increased from about $10 \%$ to $80 \%$, at an optimal rate that steadily maintains the gradient constraint active. At about $t=0.44$, the nonmonotonic load profile reaches a maximum of $90 \%$ before decreasing to $80 \%$ at $t=0.51$. This behavior is related to the optimization formulation that penalizes deviations from the reference load of $100 \%$ and may therefore lead to overshoots before the gradient constraint becomes too constraining. The overshoot that is allowed at low input penalty coefficient $\beta$ is not observed in the case of a monotonically increasing load. The dip in the temperature gradient observed at about $t=0.48$ is due to the limited degree of freedom and its amplitude decreases with an increasing discretization level. In the case of a monotonically increasing load, the gradient dip cannot be avoided and is rather independent on the discretization level. After scaling the value of the objective function is 1 for the monotonically increasing power output case and 0.95 in the non monotonic case.

\subsection{DOF Optimization of CCPP2}

The CCPP 2 model contains 28 continuous time states and 389 scalar equations. When optimizing the $2 \mathrm{DOF}$ case the input signal representing the power output was defined as non monotonic. The second input, the opening of the bypass valve, could vary from closed to fully open with a derivative in the interval $[-0.5,0.5]$. The model has been successfully optimized to full load, see results in Figure 6 where the solid trajectory represents the solution of the optimization problem.
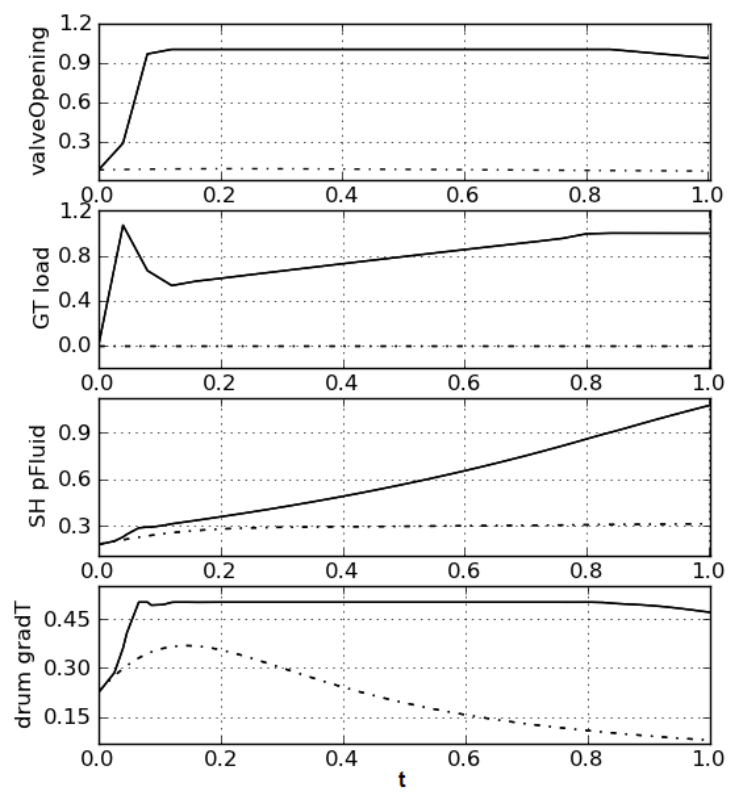

Figure 6: Optimal start-up trajectories for 2 DOF: solid (non-monotonic power output) curve. Simulated initial guess trajectories are shown by dash-dotted curves. From top: the bypass valve opening, the power output, the pressure in the superheater on the steam/water side and the temperature gradient in the wall of the drum. All results and times have been normalized.

The optimal time for the GT to reach $95 \%$ of full load is approximately $t=0.75$ when the model with 2 DOF is optimized, see Figure 6 . The temperature gradient constraint is active from $t=0.06$ and the GT load 
can not increase as rapidly as initiated after $t=0.04$. At about $t=0.12$ the GT load increases steadily at almost constant rate to not violate the temperature gradient constraint until it reaches its maximum value at $t=0.84$. The dip in the temperature gradient that was observed in the 1 DOF case is not observed. The gradient constraint is not completely active around $t=0.1$ which most likely is due to the discretization. The bypass valve is opened at $t=0$ and is fully opened at $t=0.12$, inducing that the power load can be increased more rapidly for $t<0.5$, comparing to the $1 \mathrm{DOF}$ case. After scaling the value of the objective function is 0.19 in the 2 DOF non monotonic case, and this value also includes a contribution from the $d v / d t$ term in the cost function.

The total power produced during the start-up procedure corresponds to the area under the graph of the power output. Even though the GT reaches full load later than in the $1 \mathrm{DOF}$ case, the $2 \mathrm{DOF}$ model produces more GT power during the start-up than the 1 DOF model. The objective function value corresponding to the deviation of the power output from full load is thus about $1 / 5$ of the 1 DOF model solution. This result shows the benefit of using an extra degree of freedom.

\subsection{DOF Optimization of CCPP3}

The model CCPP 3 contains 39 continuous time states and 576 scalar equations. Two different optimization problems based on CCPP3 are considered in this section.

\subsubsection{Constant Temperature Gradient Bounds}

An optimization problem based on CCPP3 with constant bounds on temperature gradients has been successfully solved, where full load is reached, see the results in Figures 7 and 8, dashed curves.

The degrees of freedom were $d u / d t$ and $d v / d t$. The GT load input $u$ was non-monotonic and the bypass valve was controlled in the optimization so that the opening of the bypass valve could vary from closed to fully open with a derivative in the interval $[-0.5,0.5]$. The optimal time for the GT to reach $95 \%$ of full load was approximately $t=0.45$, see Figure 7 .

The GT load can not increase as rapidly as initiated after $t=0.04$ since at the end time of the second block $(0.04<t \leq 0.08$, since the degree of freedom $d u / d t$ is piecewise constant) the header constraint is active. The header temperature gradient constraint is active from $t=0.08$ until $t=0.3$. The drum tempera-

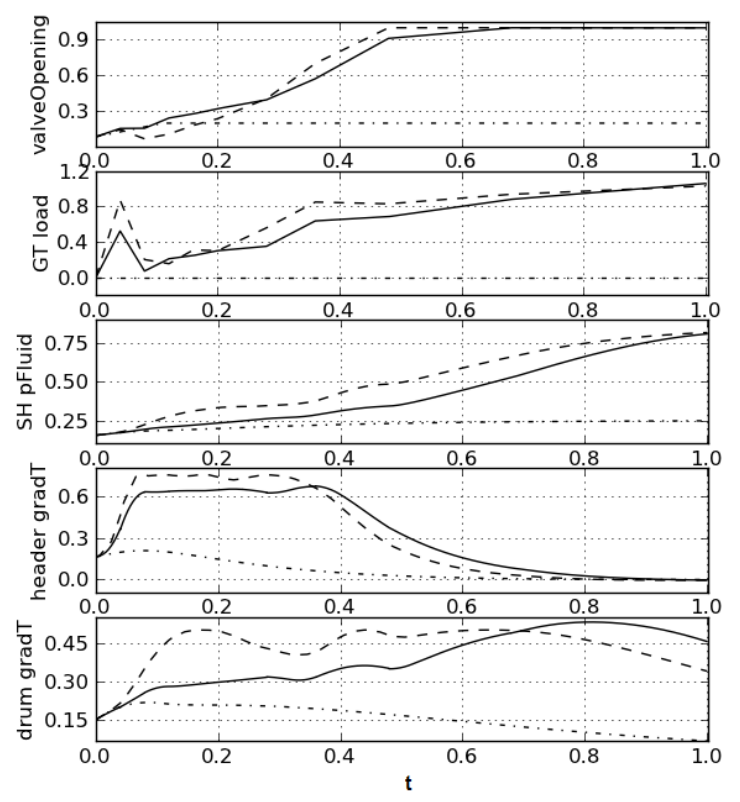

Figure 7: Optimal start-up trajectories of CCPP3. Dashed curves show results for constant temperature gradient bounds and solid curves show results for pressure dependent constraints. Simulated initial guess trajectories are shown in dash-dotted curves. From the top: bypass valve opening, GT power output, pressure in the superheater on the steam/water side, temperature gradient in the wall of the header and temperature gradient in the wall of the drum. All results and times have been normalized.

ture gradient constraint is active at different times from $t=0.16$ and it is the only active temperature gradient constraint when $t>0.3$. Around $t=0.6$ the drum temperature gradient constraint is active for the longest time sequence.

From $t=0.12$ the GT load increases with a rate that varies to not violate the header drum constraint. After $t=0.2$ the GT load increases with a steady almost constant rate until it reaches about $80 \%$ of full load at $t=0.34$. The drum temperature gradient constraint is not active during this time period. From $t=0.34$ the GT load increases at a low rate to not violate the drum temperature gradient constraint until it reaches its maximum value of 1 at $t=0.88$. The bypass valve is opened at $t=0$ and is fully opened at $t=0.48$. The valve though closes at $t=0.08$ giving a rise in the HRSG pressure and the drum temperature gradient.

After scaling the value of the objective function is 0.34 , and this value also includes a contribution from the $d v / d t$ term in the cost function. 

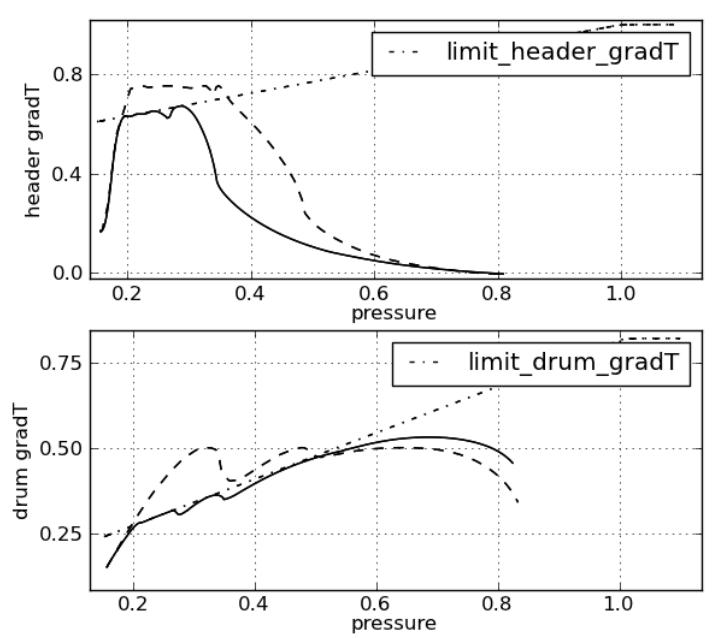

Figure 8: Optimal start-up trajectories of CCPP3. Dashed curves show results for constant temperature gradient bounds and solid curves show results for pressure dependent constraints. The dash-dotted curve shows the pressure dependent stress constraint. From the top: temperature gradient in the wall of the header as a function of pressure and the temperature gradient in the wall of the drum as a function of pressure. All results and times have been normalized.

\subsubsection{Pressure Dependent Temperature Gradient Constraint}

In Figures 7 and 8, the results for the case when applying pressure dependent temperature gradient constraints to CCPP3 are shown in solid curves.

The GT load input $u$ was non-monotonic and the bypass valve was controlled in the optimization so that the opening of the bypass valve could vary from closed to fully open with a derivative in the interval $[-0.5,0.5]$. The degrees of freedom were $d u / d t$ and $d v / d t$.

The optimal time for the GT to reach $95 \%$ of full load was approximately $t=0.56$. As in the case of constant stress bounds, the GT load can not increase as rapidly as initially after $t=0.04$, since at the end time of the second block $(0.04<t \leq 0.08$, since the degree of freedom $d u / d t$ is piecewise constant) the header constraint is active, see Figures 7 and 8 .

The header temperature gradient constraint is active from pressures $\mathrm{p}=0.19$ to $\mathrm{p}=0.25$ corresponding to the time period $t=0.07$ and until $t=0.24$. The drum temperature gradient constraint is active from pressures $p=0.2$ to $p=0.55$ corresponding to the times $t=0.09$ to $t=0.7$. The GT load is thus more constrained at lower pressures than in the previous case, see Figure 8.

The pressure dependent stress constraints allow the drum temperature gradient to attain larger values when $t>0.7$ (the pressure in the HRSG is larger than 0.55) compared to the constant constraint used in the previous case. The stress in the header is, however, more constrained in second case, which yields a lower rate of increase of the GT load as compared to the previous case. From $t=0.08$ the GT load increases with a rate that does not violate the header drum constraints. The bypass valve is opened at $t=0$ and is fully opened at $t=0.65$. The pressure is kept at low values when the temperature gradient constraints are active and the pressure can increase at a higher rate when $t>0.5$.

After scaling the value of the objective function is 0.47 and this value also includes a contribution from the $d v / d t$ term in the cost function.

\subsection{Discussion}

When starting up a power plant, the most desirable goal does not necessarily have to be to reach full load as fast as possible. To achieve as much power output as possible during the start-up procedure could be just as important. The results show how a larger amount of produced power during start-up can be achieved when adding the opening of the bypass valve as degree of freedom.

When using the pressure controller in the $1 \mathrm{DOF}$ model, it has been shown to function in a far from optimal way since the pressure is controlled so that the load cannot increase during the first $0.35 \mathrm{~s}$. The set point of the controller could be modified so that the bypass valve can be opened earlier in the start-up, lowering the pressure in the HRSG and giving the load more operational space where it does not violate the gradient constraint. When the bypass valve opening is used as a degree of freedom in the 2 DOF case the valve is opened earlier, lowering the pressure in the HRSG and allowing the load to be increased earlier. The results from the 2 DOF case produces the most power during the start-up and the benefits from using two degrees of freedom instead of one is clear.

The 2 DOF model produces more steam in an earlier phase of the start-up due to the faster ramp up of the GT load. It is though not taken into account in this paper to determine if the produced steam is of sufficient quality to start the third phase of the start-up procedure; the loading of the ST. A more complete picture of the efficiency of the start-up could be attained by modifying the objective function and adding 
more complex and thorough descriptions of possible objectives, so that the efficiency is maximized and the economical costs during the whole start-up transient is minimized. Thus the economical aspects of not only the load produced could be taken into account.

The rate of increase of the GT power load has been unlimited in all optimizations done in this paper. This and the fact that the GT power load is allowed to decrease gives a peak in the GT power load in the beginning of the start-up phase for all non-monotonic GT load cases. From an optimization point of view this is a satisfying result, since it is clear that the optimizer is trying to make the load reach its reference value as fast as possible. The GT load must though decrease to not violate the temperature gradient constraints. In actual power plants, such a fast increase in load could damage the GT or even not be physically applicable. By penalizing the use of the $d u / d t$ input such peaks could be avoided in the optimization.

When comparing the two optimization problems based on CCPP3, presented in Sections 4.4.1 and 4.4.1 respectively, it is clear that the time to reach full load is more or less the same even though in the second case there are stricter temperature gradient constraints at lower pressures comparing to the first case. The optimizer compensates this by using the full potential of the drum gradient constraint for $t>0.7$. The rate of increase of the GT load is slightly larger for second case when $t>0.7$ and it can be observed that the pressure increases at a higher rate than in first case. Even though the time to reach full load is approximately the same the profile resulting in the second case keeps the lifetime consumptions of the stressed components at a level used in actual power plant controls. The header constraint is active earlier in the start-up phase comparing to the drum constraint. This is due to that the hot exhaust gas from the GT enters the header first when the gas is of the highest temperature. The exhaust gas reaches the drum with a time delay and the exhaust gas is of lower temperature than when reaching the header. The drum consists of water in its fluid state and the gradient is therefore coupled to the pressure in the component. Steam is though decoupled from pressure and the header temperature gradient is more dependent on the GT exhaust gas temperature than the pressure. The basic stress model used in the second case uses constraints that are typically used in power plant control. Since the stress levels obtained with constant temperature gradient bounds violate these constraints, the result from the second case is the most preferable choice.
For additional background, results and discussions from this project, see [17].

\section{Summary and Conclusions}

In this paper it has been shown how a start-up procedure of a combined cycle power plant can be optimized with respect to the start-up time and the power production during start-up, using JModelica.org. The thermal stress in the heat recovery steam generator has been considered as the most limiting constraint when starting up the GT to full load, i.e. its maximum power output.

Three different optimization models have been considered; one where the load $u$ of the gas turbine is the sole degree of freedom and two where both the load $u$ and the opening $v$ of the bypass valve are degrees of freedom. Also, two different levels of model fidelities have been considered. Based on these, four optimization problems have been successfully solved where the power output has been controlled to the reference value of $100 \%$ and it has been observed that by adding the opening of the bypass valve as degree of freedom a larger amount of power during start-up is produced. In addition, it has been shown how pressure dependent stress constrains contributes to increased lifetime of critical components, which maintaining fast startups.

The models have been adapted to suit optimization purposes concerning the start-up of the GT and thus the ST has not been modeled. The next step towards achieving more realistic results could be to close the steam cycle and to include more detailed components in the model. More constraints could as well be used and additional degrees of freedom could be added. It has not been taken into consideration when it is most optimal to start the ST and if the optimization of the GT loading should take this factor into account. One improvement could thus be to find when, during the start-up procedure, the ST should be started and to determine when and how much of the steam should pass the bypass valve. Another improvement could be to include economical aspects and to minimize the fuel spent during start-up while maximizing the produced power load. The work presented in this paper is one step towards an optimal power plant control and could be used with an on-line strategy such as model predictive control. 


\section{Acknowledgments}

The German Ministry BMBF has partially funded this work (BMBF Förderkennzeichen: 01IS09029C) within the ITEA2 project OPENPROD (http://www.openprod.org). Modelon's contribution to this work was partially funded by Vinnova within the ITEA2 project OPENPROD (dnr: 2010-00068). Johan Åkesson acknowledges financial support from Lund Center for Control of Complex systems, funded by the Swedish research council.

\section{References}

[1] Casella, F. and Pretolani, F. Fast Start-up of a Combined-Cycle Power Plant: A Simulation Study with Modelica. In: Modelica Conference, pp. 3-10, Vienna, Austria, 2006.

[2] Casella, F., and Leva, A. Modelica open library for power plant simulation: design and experimental validation. In: Proceedings of $3 \mathrm{rd}$ International Modelica Conference, pp. 41-50. Linkoping, Sweden, 2003.

[3] Casella, F., Farina, M., Righetti, F., Scattolini, R., Faille, D., Davelaar, F., Tica, A., Gueguen, H. and Dumur, D. An optimization procedure of the start-up of combined cycle power plants. In: 18th IFAC World Congress, pp. 7043-7048. Milano, Italy, 2011.

[4] Casella, F., Donida, F. and Åkesson, J. Objectoriented modeling and optimal control: a case study in power plant start-up. In: 18th IFAC World Congress, pp. 9549-9554. Milano, Italy, 2011.

[5] Shirakawa, M., Nakamoto, M. and Hosaka, S. Dynamic simulation and optimization of startup processes in combined cycle power plants. In: JSME International Journal, vol. 48 (1), pp. 122128, 2005.

[6] Dassault Systemes. Dymola, http://www.3ds.com/ products/catia/portfolio/dymola, 2012, viewed 2012-06-12.

[7] Kehlhofer, R., Warner, J., Nielsen, H., Bachmann, R. Combined-Cycle Gas and Steam Turbine Power Plants, second edition, PennWell Publishing Company, 1999.
[8] Biegler, L., Cervantes, A., Wachter, A. Advances in simultaneous strategies for dynamic optimization, Chemical Engineering Science 57, pp. 575593, 2002.

[9] Modelon AB. JModelica Home Page. http://www.jmodelica.org, 2009, viewed 2012-06-12.

[10] Andersson, C., Åkesson, J., Führer, C., Gäfvert, M. Import and Export of Functional Mock-up Units in JModelica.org. In: 8th International Modelica Conference 2011. Modelica Association, 2011.

[11] Åkesson, J., Årzen, K.E., Gafvert, M., Bergdahl, T., and Tummescheit, H. Modeling and optimization with Optimica and JModelica.org languages and tools for solving large-scale dynamic optimization problems. In: Computers and Chemical Engineering, vol. 34 (11), pp. 17371749, 2010.

[12] Biegler, L. Nonlinear Programming: Concepts, Algorithms, and Applications to Chemical Processes, SIAM, 2010.

[13] Biegler, L., Wächter, A. On the Implementation of a Primal-Dual Interior Point Filter Line Search Algorithm for Large-Scale Nonlinear Programming, Mathematical Programming 106(1), pp. 25-57, 2006.

[14] Åkesson J. Languages and Tools for Optimization of Large-Scale Systems, PhD Thesis ISRN LUTFD2/TFRT-1081-SE, Regler, 2007.

[15] Modelica Association. The Modelica Association, https://www.modelica.org, 2012, viewed 2012-06-12.

[16] Bauer, O. Modelling of Two-Phase Flows with Modelica, Master's Thesis, Lund University, Department of Automatic Control, 1999.

[17] Lind, A., Sällberg, E. Optimization of the Startup Procedure of a Combined Cycle Power Plant, Master's Thesis, Lund University, Department of Automatic Control, 2012. 
